\title{
Individual Differences Predict Patterns in Spontaneous Involuntary Musical Imagery
}

Daniel Müllensiefen \& Joshua Fry Goldsmiths, University of London, London, United Kingdom

Rhiannon Jones University of Winchester, Winchester, United Kingdom

SAGAR JILKA Imperial College, London, United Kingdom

Lauren STEWart Goldsmiths, University of London, London, United Kingdom

Victoria J Williamson

Lucerne University of Applied Sciences and Arts, Luzern, Switzerland and University of Sheffield, Sheffield, UK

INVOLUNTARY MUSICAL IMAGERY (INMI) DESCRIBES the everyday phenomenon of having a tune stuck in the head. Research has established the ubiquity of this form of spontaneous cognition but the predictive role of individual differences is still debated. This study examines the impact of everyday musical behaviors and subclinical obsessive compulsive attributes on INMI experiences. In total 1,536 participants completed three online questionnaires; a novel inventory of musical behavior and INMI, and a standardized obsessive compulsion (OC) inventory. Exploratory factor analysis $(N=512)$ and structural equation modelling $(N=$ $1,024)$ were applied. Everyday singing and music listening positively predict length and frequency of reported INMI episodes, respectively. No relationships were found with musical training. High OC was positively related to INMI frequency and disturbance, but only indirectly to INMI episode length and unpleasantness. The identified contributory factors of INMI experiences are discussed in the context of musical memory and spontaneous mental activity.

Received: January 30, 2013, accepted June 25, 2013.

Key words: involuntary musical imagery, "earworms," spontaneous cognition, obsessive-compulsive behaviors, mental imagery
$\mathrm{E}$ VERYDAY TASKS ARE FREQUENTLY ACCOMPAnied by task-independent thoughts or "spontaneous cognitions" (Christoff, Ream, \& Gabrieli, 2004; Klinger \& Cox, 1987; McVay, Kane, \& Kwapil, 2009). Up to $30-40 \%$ of thoughts in daily life may be unrelated to the task at hand (Hurlburt, 1979; Kane et al., 2007; McVay et al., 2009). These mental experiences include involuntary autobiographical memories (Bernsten, 1998, 2010), involuntary semantic memories (Kvavilashvili \& Mandler, 2004) and primed thoughts (Tulving \& Schacter, 1990).

The tendency towards spontaneous cognitions is a stable cognitive characteristic, "representing an individual difference that is reliable across time, activities, and contexts" (McVay et al., 2009, p. 861). However, the reasons for interindividual differences in spontaneous cognitions are not yet well understood. This situation is due partly to the empirical difficulties associated with measurement, as spontaneous cognitions are unpredictable, transient, and ephemeral. However, one pervasive, ubiquitous, and classifiable form of spontaneous cognition holds promise as a tool for determining the substrates of spontaneous cognition: Involuntary Musical Imagery (INMI).

INMI describes the conscious, internal experience of a musical excerpt in the absence of an external stimulus, which then goes on to repeat outside of conscious will or control (Liikkanen, 2012; Williamson et al., 2012). The colloquial term associated with INMI is "earworms" (a translation from the German "Ohrwurm"); other terms include "brain worms," "sticky music" (Sacks, 2007), and "spontaneous musical imagery" (Wammes \& Barušs, 2009). In the present study we draw a distinction between INMI and more vivid and externally attributed musical imagery experiences associated with psychosis or pathological musical hallucinations (Mahendran, 2007; Sanchez et al., 2011; Warner \& Aziz, 2005).

INMI is a ubiquitous, everyday experience. Liikkanen (2012) surveyed over 11,000 Finnish internet users and found that $33.2 \%$ experienced INMI every day, with $26.2 \%$ reporting that episodes occurred several times a day. Beaman and Williams (2010) reported that $88.2 \%$ of people had INMI experiences that lasted hours or longer. Finally, Halpern and Bartlett (2011) conducted a survey and diary studies where participants recorded

Music Perception, volume 31, issue 4, pp. 323-338, issn 0730-7829, electronic issn 1533-8312. C 2014 by the Regents of the university of California All RIGHTS RESERVED. PLEASE DIRECT ALL REQUESTS FOR PERMISSION TO PHOTOCOPY OR REPRODUCE ARTICLE CONTENT THROUGH THE UNIVERSITY OF CALIFORNIA PRESS'S RIGHTS AND PERMISSIONS WEBSITE, HTTP://WWW.UCPRESSJOURNALS.COM/REPRINTINFO.ASP. DOI: 10.1525/MP.2014.31.4.323 
INMI over the course of one and two weeks, and found INMI to be ubiquitous both in terms of the high proportion of experiences and the frequency with which the majority of people were affected (median 7 experiences per week). Taken together, these results confirm the pervasive, universal, and persistent nature of INMI experiences.

Prior evidence suggests that individual differences might be linked to certain attributes of INMI (e.g., frequency), indicating that INMI may have the same stable context invariant attributes as other spontaneous cognitions. Beaman and Williams (2010) reported that people who rated music as important to them ${ }^{1}$ experienced INMI that was more frequent, longer, and more troubling. Liikkanen (2012) reported small relationships (Cohen's $h$ between .05 and .36) with INMI frequency and the amount of time spent listening to music, level of self-reported musicality, and the amount of time spent practicing a musical instrument. ${ }^{2}$ These findings suggest that everyday musical engagement predicts aspects of INMI experiences more reliably than other basic individual differences such as age or gender. One potential explanation for these findings is that regular activation of neural processes associated with music listening, many of which overlap with activity seen in purposeful musical imagery (Halpern \& Zatorre, 1999; Hickok, Buchsbaum, Humphries, \& Muftuler, 2003; Kleber, Birbaumer, Veit, Trevorrow, \& Lotze, 2007; Kraemer Macrae, Green, \& Kelley, 2005; Zatorre \& Halpern, 2005), may drive individual characteristics in INMI.

The first aim of the present study was to extend existing research in order to examine the causal effects of a wider range of facets of everyday musical engagement upon characteristics of INMI episodes. In addition to more traditional measures (amount of training), we assessed various aspects of everyday musical behaviors that have yet to be examined with relation to INMI. Musical engagement includes many factors such as commitment to music, the experience of positive psychotropic effects in response to listening, and reactive musical behavior such as singing (Werner, Swope, \& Heide, 2006) as well as current music listening attitudes or the importance of music during childhood (Cuddy, Balkwill, Peretz, \& Holden, 2005). These factors can have an independent influence on complex cognitive processes such as verbal learning (Chin \& Rickard, 2010), so it is important to disentangle these effects and determine the extent to which they independently influence aspects of INMI.

\footnotetext{
${ }^{1}$ Question was based on a yes or no response.

${ }^{2}$ Listed in increasing order of correlation strength.
}

Individual differences in traits that relate to thinking styles may also influence INMI experiences; in particular, traits that predict that an individual will experience an increase in thought incursions. Wammes and Barušs (2009) found an association between participants who scored high on "Transliminality" and more persistent and distracting INMI. Transliminality is a construct that represents the "degree of permeability of a psychological membrane" (Wammes \& Barušs, 2009 , p. 40), the likelihood that thoughts from the subconscious will be realized in the conscious mind. Floridou, Williamson, and Müllensiefen (2012) also noted an association between neuroticism and several aspects of INMI experiences. Most notably, neuroticism correlated negatively with the subjective pleasantness of INMI experiences and positively with the tendency to worry about eaworms, the length of earworm episodes, and their potential to interfere with other activities.

Individual difference in thought incursions can also be characterized by obsessive compulsion (OC) attributes. A link between INMI and OC has intuitive appeal, since frequent and negative thought intrusions are one of the defining symptoms of obsessive compulsive disorder (OCD; DSM-IV), and INMI can be both negative and intrusive (Beaman \& Williams, 2010; Brown, 2006; Liikkanen, 2008). Like OC thought patterns, INMI can exhibit a cyclic repeating pattern, sometimes over several hours (Brown, 2006). Levitin (2006) suggested that individuals with OCD are more likely to experience INMI, while Sacks (2007) proposed a similar continuum related to OCD and Tourette's syndrome. There are, however, only two reported case studies of patients with musical imagery obsessions (Praharaj et al., 2009; Zungu-Dirwayi, Hugo, van Heerden, \& Stein, 1999), so the relationship between OCD and INMI has yet to be reliably established.

Measurement of OC in a subclinical population can provide a valid analogue to that of a clinical OCD population; OC occurs on a continuum in the general population (Gibbs, 1996), and has shown to be qualitatively indistinct from clinical symptoms, differing only in severity (Frost, Steketee, Cohn, \& Griess, 1994; Gershunny \& Sher, 1995; MacDonald \& de Silva, 1999; Sternberger \& Burns, 1990; Wu \& Watson, 2002). Studies have also reported similar relationships between subclinical groups compared to patients with regards to deficits in neuropsychological (Mataix-Cols et al., 1999; Sher, Frost, \& Otto, 1983; Sher, Mann, \& Frost, 1984), memory function (Rubenstein, Peynircioğlu, Chambless, \& Piggot, 1993), and similarities in task-related brain activity (Cottraux et al., 1996; Mataix-Cols et al., 2003). 
The second aim of the present study is to determine whether subclinical OC attributes relate to INMI experiences. Research has shown that frequency of intrusive thoughts correlates with measures of subclinical OC attributes (García-Soriano, Belloch, Morillo, \& Clark, 2011), and that the frequency of neutral as well as negative intrusions is elevated in OC individuals (Tolin, Abramowitz, Przeworski, \& Foa, 2002). Individuals with OCD and high subclinical OC attributes also report increased attempts to suppress intrusive thoughts (Freeston \& Ladouceur, 1997; Wegner \& Zanakos, 1994). These attempts often cause a paradoxical increase in the frequency of the intrusion (Salkovskis \& Campbell, 1994; Wegner, Schneider, Carter, \& White, 1987; however, see Purdon, 2004). According to Wegner et al. (1987) this paradoxical increase can occur during suppression (the immediate enhancement effect) or after suppression attempts have ceased (the rebound effect), which can be seen as analogous to increased INMI length and frequency, respectively, in the current study. Therefore, related to hypothesis two, we expect that higher subclinical OC individuals will report more attempts to suppress INMI episodes, will find these episodes harder to control, and therefore experience longer and/or more frequent INMI episodes.

The proposed link between subclinical OC and INMI in the present paper partially challenges how INMI experiences have been categorized in the previous literature. Beaman and Williams (2010) speculated that INMI differs from "pathologically obsessive thought[s] which, by definition, [are] distressing" (p. 12) on the grounds that the majority of INMI is pleasant (Halpern \& Bartlett, 2011) and that the recurrence rate of INMI episodes is low. While these arguments represent a reasonable counter to an association between subclinical OC and INMI, it would be premature to dismiss the idea. First, the samples in Halpern and Bartlett (2011) and Beaman and Williams (2010) were small and unlikely to contain sufficient variation in subclinical OC traits. Secondly, neither study provided any measure of OC but instead noted only post hoc related measures of INMI recurrence and pleasantness. The final aim of the present study is therefore to assess for the first time the relationship between INMI and subclinical OC.

\section{HYPOTHESES}

We study the ubiquitous phenomenon of INMI in a large cohort from the general population in order to examine whether multiple characteristics of INMI, including frequency, intrusiveness, controllability, length, and pleasantness, can be ascribed to individual differences in either 1) everyday music engagement, and/or 2) subclinical OC attributes.

Our first group of hypotheses is that individuals with more extensive musical engagement, as measured by everyday musical behaviors, will experience more frequent episodes of INMI (Beaman \& Williams, 2010; Liikkanen, 2012) that are longer in length and more troubling (Beaman \& Williams, 2010).

Our second group of hypotheses is that higher OC individuals will experience more frequent INMI that interferes more with their everyday life. We also expect that these individuals will report more attempts to suppress INMI, find it harder to control, and experience longer INMI episodes. Hypotheses relating to subclinical OC may interact with whether individuals view their INMI as unpleasant or disturbing experiences (Purdon, 2004). We will thus pay special attention to this mechanism within the statistical analysis.

\section{Method}

\section{PARTICIPANTS}

Data from 1,787 individuals was gathered via an online survey. We excluded 197 individuals who reported hearing problems and 54 participants who did not complete the necessary questions for the present study. This left 1,536 participants ( $58.1 \%$ women, $41.4 \%$ men, $0.5 \%$ gender undisclosed) for subsequent analysis. The mean age of the participant sample was $34.2(S D=12.6$, age range $=12-75$ years) .

\section{MATERIALS}

The online survey was hosted at http://earwormery.com and promoted through various media including radio and online features across the BBC network, as well as networks in the USA and Australia, emails to universities in the UK, USA, Europe, and Australia, promotion across Facebook, Twitter, and other social media, and through leafleting in London, York, and Birmingham (Williamson et al., 2012).

Three questionnaires from the online survey were relevant to the present study. The first was The Obsessive Compulsive Inventory - Revised (OCI-R; Foa et al., 2002). The second assessed everyday musical behaviors and expertise as a musician or music professional. Because of the lack of standardized psychometric instruments in this area at the time of testing ${ }^{3}$ we compiled a single questionnaire comprising 16 items that assessed

\footnotetext{
${ }^{3}$ This research was carried out before the Goldsmiths Musical Sophistication Index (Müllensiefen, Gingras, Musil, \& Stewart, 2014) was available as a standardized self-report instrument.
} 
musical behavior and engagement as well as different aspects of musical expertise such as music playing and practice habits (henceforth, the musical behavior questionnaire). The final questionnaire (henceforth, the INMI questionnaire) assessed all relevant parameters of INMI episodes for the present hypotheses; length, frequency, intrusiveness, pleasantness, and control strategies. Again, because of the lack of standardized materials this was a novel self-report instrument, based on existing questions by Liikkanen (2012) and Beaman and Williams (2010). All questionnaires can be found in Appendix A.

\section{PROCEDURE}

All questionnaires were completed online between May 2010 and March 2011. Participants entering between May and September 2010 were entered a prize draw for $£ 100$. Users took, on average, between 15 and 20 minutes to complete all three questionnaires.

\section{DESIGN}

The analysis of this study is broken down into two parts. Because the musical behavior and INMI questionnaires were new, we first investigated their latent structure using exploratory factor analysis. This reduced the dimensionality of the data and exposed the factorial structure of the respective domains. In the second stage of the analysis these latent factor structures were compared using confirmatory analysis and then examined with structural equation modelling (see Loehlin, 2004, for a good introduction) in relation to the proffered hypotheses concerning the relationships between musical behavior and subclinical OC on INMI. In order not to over-fit the data, one third of the sample (512 participants) was used for the exploratory analysis and the other two thirds of the sample (1024 participants) for the confirmatory analysis.

\section{Results}

Summary statistics for all items of the Musical Behavior and the INMI questionnaire as well as for the OCI-R subscales are given in Appendix B Table 1. The means of the six OCI-R subscales (Washing $=1.07$, Neutralizing $=1.41$, Checking $=2.33$, Hoarding $=3.77$, Obsessing $=2.76$, Ordering $=3.13$ ) all fall below the clinical thresholds (Washing $=3$, Neutralizing $=3$, Checking $=5$, Hoarding $=5$, Obsessing $=5$, Ordering = 7) that Gönner, Hahn, Leonhart, Ecker, and Limbacher (2009) found for an optimal (sensitivity and specificity ranging from .83 to 1.00 and from .76 to .92 ) discrimination between clinical and nonclinical OC symptoms. Hence, the subscale means from the current dataset confirm that our sample represents a nonclinical population.

\section{EXPLORATORY FACTOR ANALYSES}

Musical behavior and expertise. This questionnaire comprised sixteen items that assessed attentive and background listening, number of concerts attended, self-assessed singing ability, and frequency of singalong behavior, as well as the importance of music, self-defined levels of musical competence, type and extended of music training on an instrument and other skilled musical activities, and the possession of absolute pitch. The correlational structure of all sixteen items is shown in Figure 1.

In order to explore the latent structure of musical behavior, items of limited use for discriminating between participants were removed. Adapting recommendations by Comrey (1988) and Clark and Watson (1995), any item where $85 \%$ or more of the responses fell into a single category was removed, which affected two items-absolute pitch possession and professional music activities-other than playing music (music producer, piano tuner, etc.).

A series of maximum-likelihood principal factor analyses were then carried out on the correlation matrix of the remaining fourteen items, extracting one to eight factors while testing for each solution. The aim was to determine whether the number of factors was sufficient to describe the data against the alternative hypothesis that the true covariance in the population would not be constrained by the factors. This test indicated that six factors might be an upper bound for the number of factors to be extracted, $\chi^{2}(22)=23.96, p=.35$.

The factor loadings of the fourteen items on these six factors were inspected after varimax rotation. The first four factors had high loadings from several items while factors five and six only had one high loading item each and were therefore not measuring any latent concepts. The visual inspection of the screeplot of eigenvalues gave ambiguous results so we also conducted a "parallel analysis" (Horn, 1965) comparing the eigenvalues extracted from our data to eigenvalues of a random data set of the same size. This parallel analysis confirmed that four factors should be extracted. It was therefore decided to compute a final four-factor solution for the Musical Behavior questionnaire that explained $52.6 \%$ of the variance among the variables. The solution is shown in Table 1.

Involuntary musical imagery. The INMI questionnaire contained seven items regarding the length and frequency 


\section{Correlation Matrix of Musical Behavior Variables}

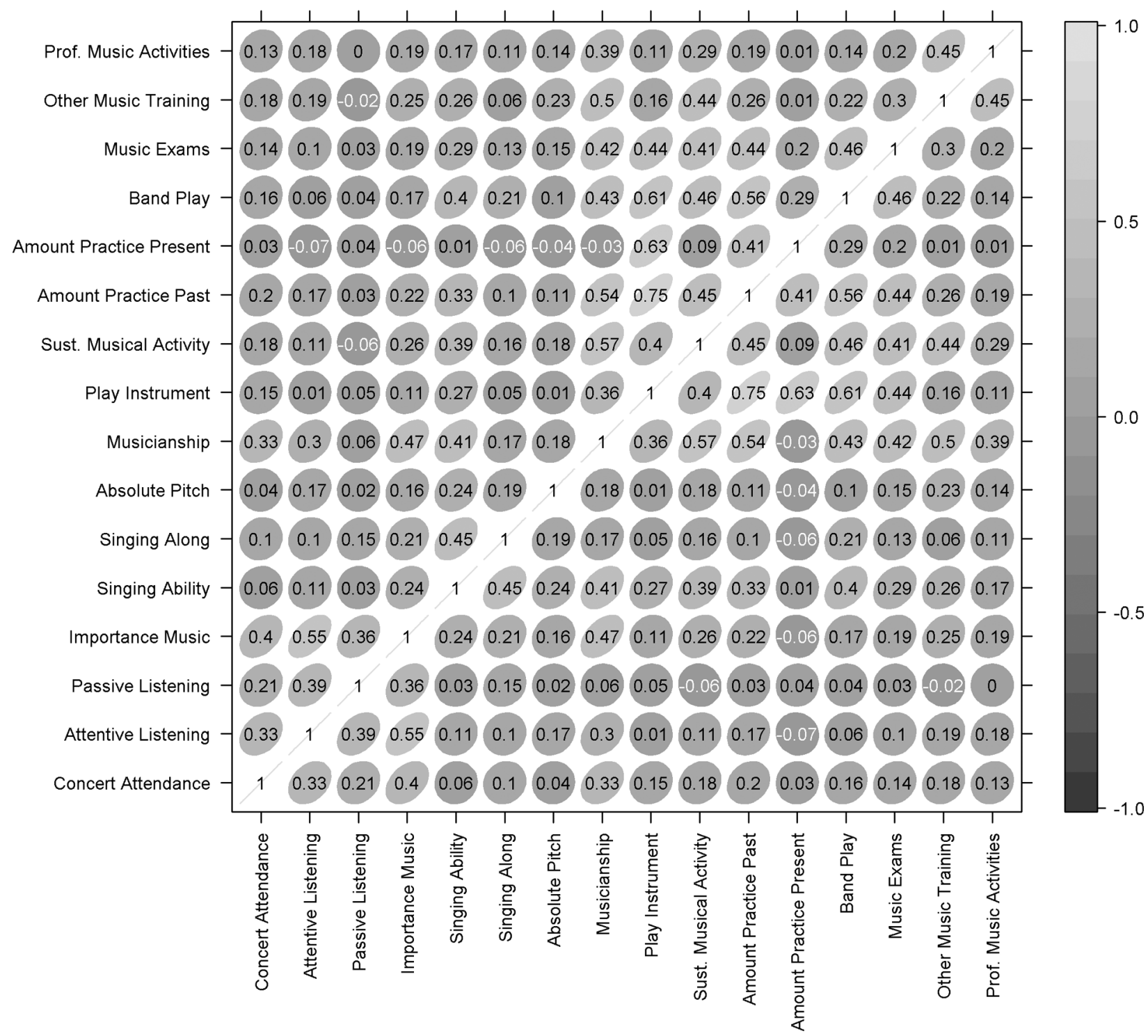

FIGURE 1. Correlation structure of items from musical behavior questionnaire on exploratory data set $(n=512)$. Shapes represent absolute magnitude of correlations: Ellipsoid shapes represent stronger absolute correlations compared to circular shapes. Gray scale represents signed value of correlation from -1 (dark gray) to 1 (light gray). Correlations with a value of $r>|.09|$ are significant at the $5 \%$ level after correcting for multiple comparisons using Holm's (1979) correction procedure.

of INMI episodes, their subjective unpleasantness, the frequency with which an individual tries to get actively rid of their INMI, the effort necessary for controlling them, interference with other tasks, and the degree to which an individual feels their INMI experiences are worrisome. Summary statistics for all questionnaire items are given in Appendix B Table 1. The correlational structure is given in Figure 2.
A series of factor analyses were carried out in line with the procedure described in the previous section and established three factors that could be considered as upper bound for the factorial structure of the data, $\chi^{2}(3)=2.18, p=.54$. A parallel analysis also suggested a three-factor solution. However, the three-factor solution explained only $38.5 \%$ of the variance and only the first factor had an eigenvalue $>1$, with subsequent factors 
TABLE 1. Results of Exploratory Factor Analysis on Items from Musical Behavior Questionnaire.

\begin{tabular}{lclc}
\hline Factor Name & Variance Explained & Items & Factor Loading \\
\hline Musical Practice & $18.7 \%$ & Play an Instrument & .91 \\
& & Amount of Instrumental Practice (Past) & .75 \\
& & Amount of Instrumental Practice (Present) & .77 \\
& & Play in Band / Ensemble & .57 \\
& & Taken Music Exams & .42 \\
\hline Music Professionalism & $14.8 \%$ & Self-rated Musicianship & .81 \\
& & Years of Sustained Musical Activity & .64 \\
& & Musical Training Beyond Instrument & .52 \\
\hline Listening Engagement & $12.5 \%$ & Amount of Attentive Listening & .80 \\
& & Importance of Music in Life & .71 \\
& & Amount of Passive Listening & .57 \\
Singing & & Concert Attendance & .39 \\
& & Self-rated Singing Ability & .56 \\
\hline
\end{tabular}

Note. The final four-factor solution for the musical behavior questionnaire. Table shows given factor names, amount of variance accounted for by each individual factor and the original questionnaire items together with the loading for the factor that each item loaded highest on.

having eigenvalues of less than .5. This was evident from a screeplot that indicated an "elbow" at the second factor (suggesting a one-factor solution). In addition, inspection of the factor structure revealed that several items loaded almost equally highly on more than one factor and that at least one factor had no high loading items at all. It was therefore decided to exclude the three items with the highest uniqueness scores (INMI frequency, INMI length, INMI unpleasantness) from the factor analysis and to treat them as distinct variables for the remainder of this analysis.

Repeating the factor analysis with the four remaining items (using varimax rotation) revealed one common INMI factor, $\chi^{2}(2)=0.19, p=.91$. All remaining variables loaded with values of at least .48 on this factor, and because all four items measured aspects of subjective valence or how disturbing INMI episodes were considered, we termed this common latent factor INMI Disturbance. The loading of the four items on the common factor are given in Table 2.

\section{CONFIRMATORY ANALYSIS}

Data from the remaining 1,024 participants were now employed to compare the latent factor structures for the three scales (OC, Musical Behavior, and INMI) and to directly investigate the hypotheses between the scales using structural equation modelling.

To get an initial overview of the correlation structure, factor scores for the four latent factors of Musical Behavior (Musical Practice, Music Professionalism,
Listening Engagement, and Singing) as well as for the INMI latent factor of INMI disturbance were computed using Bartlett's method of weighted least-squares (DiStefano, Zhu, \& Mîndrilă, 2009; Everitt \& Hothorn, 2011). A correlation table was computed between these and the existing factors from the three different scales (OC, Musical Behavior, and INMI). The graph of the total correlation matrix revealed several positive but minor correlations between OC-subscales and the disturbance and frequency of INMI episodes (Figure 3). INMI frequency was mildly correlated with Singing $(r=.17)$ and even slightly less with Music Listening $(\mathrm{r}=.15)$. INMI length had a low correlation only with Singing $(r=.12)$. All other correlations between INMI variables and musical behaviors as well as OC-factors were negligibly small. As the graph shows, the strongest correlations were observed among the six OCI-R subscales (range $=.26$ to .54 ) which is in line with previous studies that also identified substantial interfactor correlations of the OCI-R (range $=.23$ to .51 , Foa et al., 2002).

Before proceeding further with direct hypothesis testing it was important to confirm the measurement model of the two new scales and to identify the underlying structural relationships between INMI, OC, and Music Behavior. A $27 \times 27$ correlation matrix was computed using the 14 observed variables of Music Behavior, the 7 INMI variables, and the 6 OC subscale scores. This correlation matrix served as input to a structural equation model in order to test the structural relations between the latent constructs posited in the experimental hypotheses. 


\section{Correlation Matrix of INMI Variables}

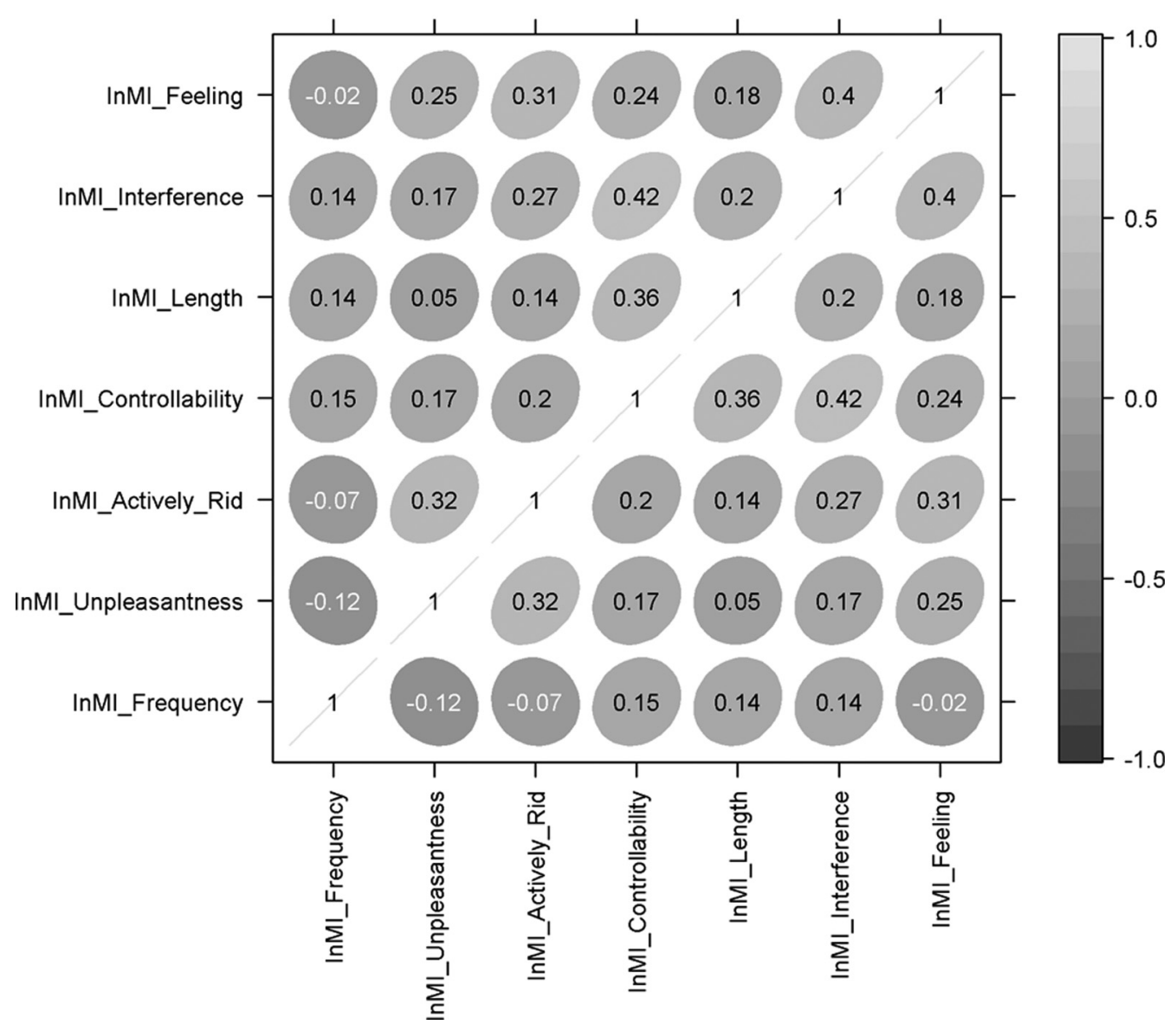

FIGURE 2. Correlation structure of items from the INMI questionnaire on exploratory data set $(n=512)$. Correlation coefficients within the diagram are multiplied by 100. Gray scale and shape coding matches that in Fig. 1. Correlations with a value of $r>|.09|$ are significant at the $5 \%$ level after correcting for multiple comparisons using Holm's (1979) correction procedure.

Driven by the theoretical considerations and prior assumptions outlined above, the model specified a positive influence of the four latent factors of Musical Behavior on frequency, length, unpleasantness, and disturbance of INMI experiences (Hypothesis Group 1) as well as a positive influence of higher OC traits on INMI frequency, unpleasantness, and disturbance, which in turn lead to longer INMI episodes (Hypothesis Group 2).

In order to find a structural equation model from a correlation matrix, we used the package 'sem; Structural Equation Models' (Fox, 2006), which employs a full information maximum-likelihood criterion and a nonlinear minimization algorithm. The initial model included paths from all four latent musical factors and the overall OCD score to the latent factor INMI_Disturbance as well as the other three OC variables (length, frequency, and unpleasantness). This full model is depicted in Figure A1 in the Appendix. Several goodness-of-fit statistics indicated an acceptable overall fit to the data, $\chi^{2}(294)=1465.00, p<.001$; Bentler's Comparative Fit Index $(\mathrm{CFI})=.86$; Tucker-Lewis-Index $(\mathrm{TLI})=.83$; RMSEA Index $=.06$; $\mathrm{SRMR}=.06$; $\mathrm{BIC}=$ 2040.1. All paths from observed variables to latent factors were highly significant and possessed generally large standardized estimates (between .41 and .96), confirming the measurement models for the Musical Behavior and INMI scales that were derived in the exploratory phase.

Finally, we removed all nonsignificant paths and observed variables associated with the latent factors Music_Practice and Music_Professional, which did not have any significant paths with any of the INMI variables. The fit of the new model was substantially better as a result, $\chi^{2}(145)=573.00, p<.001$; Bentler's Comparative Fit 
TABLE 2. Results of Exploratory Factor Analysis on Items from INMI Questionnaire.

\begin{tabular}{lclr}
\hline Factor Name & Variance Explained & Items & Factor Loading \\
\hline INMI & $30.57 \%$ & Interference of INMI Experiences & .598 \\
& & Feeling worried about INMI Experiences & .582 \\
& & Attempts to Get Rid of INMI Experiences & .537 \\
& Effort to Control INMI Experiences & .484 \\
\hline
\end{tabular}

Note. Factor loadings of four items from the INMI questionnaire onto the common INMI factor.

\section{Correlation Matrix of Musical Behavior, INMI and OCD Variables}

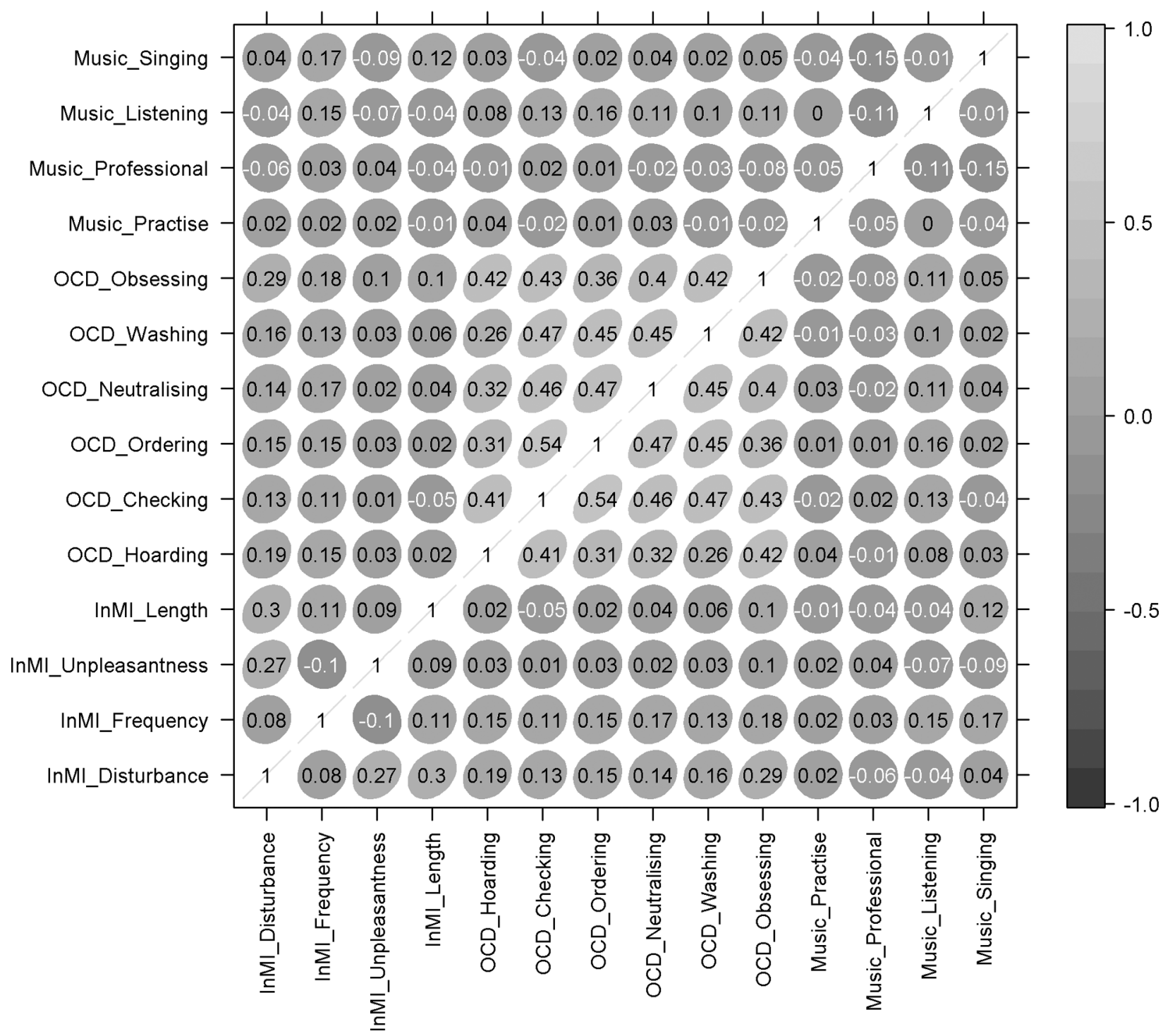

FIGURE 3. Correlation structure of items and subscales from musical behavior questionnaire, OCI-R, and INMI questionnaire on confirmatory data set ( $n$ $=1024)$. Correlation coefficients within the diagram are multiplied by 100 . Gray scale and shape coding matches that in Figure 1 . Correlations with a value of $r>|.12|$ are significant at the $5 \%$ level after correcting for multiple comparisons using Holm's (1979) correction procedure. 


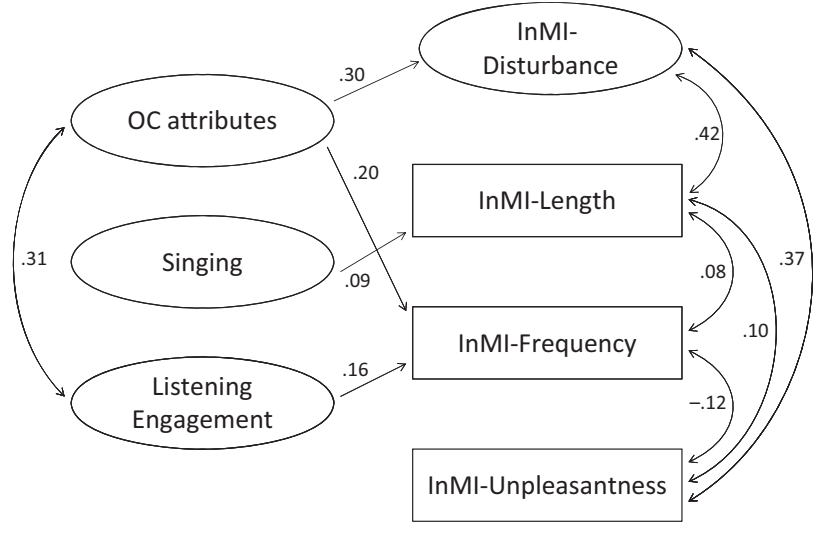

FIGURE 4. Graphical representation of the structural equation model. In accordance with common conventions, latent variables are given inside circles and observed variables are placed in rectangles. Single headed arrows between different variables represent regression coefficients and double-headed arrows within the same variable are correlation coefficients.

Index $(\mathrm{CFI})=.89 ;$ Tucker-Lewis-Index $(\mathrm{TLI})=.87$; RMSEA Index $=.05 ;$ SRMR $=.06 ; \mathrm{BIC}=89$, and subsequent testing with model modification indices (Saris, Satorra, \& Sorbom, 1987; Sorbom, 1989) did not suggest the addition of any new paths from Musical Behavior or OC factors to any of the INMI variables. The final structural equation model is depicted in Figure 4.

In relation to Hypothesis Group One, the final model demonstrates that only the latent constructs Singing and Listening had a significant effect on INMI experiences. Participants scoring higher on Singing reported their INMI to be longer (regression coefficient estimate = .09) and increased Listening scores influenced the frequency by which INMI episodes occurred (regression coefficient estimate $=.16$ ). In addition, the model includes several significant interfactor correlations and variables related to the same construct (i.e., correlation between Singing and Listening as well as intercorrelations among the four different INMI variables). In particular, the negative correlation $(r=-.12)$ between INMI frequency and INMI unpleasantness is worth noting because it suggests a tendency for individuals who experience more frequent INMI episodes to find them more pleasant.

In relation to hypothesis group two, subclinical OC has a mild positive influence on INMI frequency (standardized regression coefficient estimate $=.20$ ) and a stronger positive relation with the latent factor INMI disturbance (regression coefficient estimate $=.30$ ) that represents, among other variables, the urge to get rid of an INMI experience and the effort needed to expunge it. In turn, higher INMI disturbance co-varies with longer
INMI experiences (correlation coefficient $r=.42$ ). However, higher OC does not lead to longer INMI episodes; as predicted, this relationship is mediated by the degree to which the INMI is rated as disturbing. Also, according to the model there is no direct link between subclinical OC score and the unpleasantness of INMI episodes.

\section{Discussion}

Involuntary Musical Imagery (INMI) is a ubiquitous and regular experience (Liikkanen, 2012; Williamson et al., 2012). Previous research has revealed that individual differences can predict aspects of INMI experiences, including musical behaviors (Beaman \& Williams, 2010; Liikkanen, 2012) and thought patterns (Floridou et al., 2012; Wammes \& Barušs, 2009). This paper presents the first comprehensive statistical model to quantify relations between a wide range of everyday musical behaviors, subclinical OC attributes, and INMI characteristics.

Our first group of hypotheses stated that individuals with more extensive musical engagement, as measured by everyday musical behaviors, would experience more frequent episodes of INMI (Beaman \& Williams, 2010; Liikkanen, 2012), that were longer in length and more troubling (Beaman \& Williams, 2010). In the present analysis only the musical factors Singing and Listening were found to act upon INMI; Singing increased the reported length of INMI episodes and Listening increased their frequency of occurrence. The amount of self reported musical practice and music professionalism did not affect any aspect of INMI experiences.

These results partially confirm findings from Liikkanen (2012) who reported a relationship between INMI frequency and both music listening activity and portable music player use. Liikkanen (2012) also reported low level correlations with musical practice and self-assessed musicality that the present study did not find. However, it is important to note that the associations reported in Liikkanen (2012) were based on a very large sample (nearly 12,000), were small by conventional effect size estimates (.11 to .27 ), and that hypothesized relations between variables were not directed. Furthermore, the measurement inventories varied between studies and hence it is difficult to compare them directly. Despite these limitations, there is now converging supportive evidence that everyday music listening is associated with more frequent INMI experiences.

What might explain the association between music listening and INMI? One theory is that a significant proportion of INMI comprises activations of recently 
experienced musical memories. ${ }^{4}$ People who listen to a lot of music will likely activate their musical memories more regularly and recently compared to those who chose to listen less, rendering those memories primed to involuntary reactivation that is either spontaneous or linked by spreading activation through a related semantic network (Anderson, 1983; Collins \& Loftus, 1975; Collins \& Quillian, 1969). This theory is supported by evidence that recent and repeated exposure to music is a common situational antecedent to INMI (Williamson et al., 2012).

Not all INMI characteristics can be predicted by music listening. The present study is the first to identify singing as an important and independent musical behavior related to the subjective length of INMI. This finding contributes to the literature by specifying the potential cause of a more general relationship between musical activity and INMI that was initially identified by Liikkanen (2012) and Beaman and Williams (2010). One theory is that regular activation of neural processes associated with voluntary singing (including subvocal) causes involuntary musical imagery experiences to be longer. Such neural areas are already known to overlap with the activity seen in purposeful musical imagery (Halpern \& Zatorre, 1999; Hickok et al., 2003; Kleber et al., 2007; Kraemer et al., 2005; Zatorre \& Halpern, 2005).

The second group of hypotheses for the present study related to predicted associations between subclinical OC and INMI, which would support a theoretical link between OCD and INMI postulated by Sacks (2007) and Levitin (2006). The present model suggests that higher subclinical OC is related to some but not all measures of INMI experiences. Higher OC attributes predict an increase in INMI frequency while also acting on the subjective disturbance of these episodes: individuals with high OC report that INMI episodes are more worrisome, interfere more, and are harder to control (suppress). These individuals also make more attempts to get rid of their INMI. In turn, higher INMI disturbance is associated with longer INMI episodes $(r=.42)$ and higher INMI unpleasantness $(r=.37)$.

It should be noted that the relationships above come from retrospective self report measures and therefore may at least partly reflect an increased tendency to notice or become preoccupied by INMI. Future studies can determine whether the identified patterns remain the same when individuals INMI experiences are measured by more time sensitive methods such as Experience Sampling Methodology. Fundamentally, however, the reports we obtained reflect how different individuals conceptualize their INMI episodes.

This dependent chain mechanism between subjective disturbance (including attempts at suppression) and length of INMI is in line with our hypotheses and reports from OCD and thought-suppression literature where high efforts to suppress unwanted thoughts lead to an increase in these unwanted cognitions (Wegner et al., 1987). This paradoxical increase occurs during thought suppression (the immediate enhancement effect) and after suppression attempts have ceased (the rebound effect). In the current analysis, increased length of an INMI episode is akin to the immediate enhancement effect, as suppression attempts occur concurrently. A link between INMI disturbance and increased frequency of episodes would be akin to the rebound effect, as this refers to new INMI episodes temporally separated from the initial suppression. According to the structural model, INMI disturbance and INMI frequency have no significant direct relationship. As such, our model supports the idea that a greater immediate enhancement effect as a result of suppression in high OC individuals leads to an increase in INMI (Salkovskis \& Campbell, 1994; Tolin et al., 2002).

The present study has demonstrated that structural equation modelling techniques can be employed to illuminate how individual differences in everyday behavior and thinking style are associated with a prevalent form of spontaneous cognition; INMI. Characteristics of INMI experiences are associated with everyday singing and music listening behaviors. The valence with which these spontaneous cognitions are experienced are then partially influenced by individual differences (in OC), which in turn contribute to perceptions of INMI frequency and, indirectly, to the length of episodes. In terms of future research avenues, it would be interesting to investigate whether this general pattern of structural relations influencing INMI experiences-which can be summarized as "individual difference in thinking patterns + repeated everyday behavior" - also holds true for other forms of spontaneous cognitions such as intrusive thoughts or Eureka moments.

\section{Author Note}

Correspondence concerning this article should be addressed to Daniel Müllensiefen, Department of Psychlogy, Goldsmiths, University of London, London SE14 6NW, United Kindgom. E-mail: d.mullensiefen@gold.ac.uk

\footnotetext{
${ }^{4}$ We are grateful to our action editor for this suggestion.
} 


\section{References}

Anderson, J. R. (1983). A spreading activation theory of memory. Journal of Verbal Learning and Verbal Behavior, 22, 261-295.

Beaman, C. P., \& Williams, T. I. (2010). Earworms (stuck song syndrome): Towards a natural history of intrusive thoughts. British Journal of Psychology, 101, 637-653.

Bernsten, D. (1998). Voluntary and involuntary access to autobiographical memory. Memory, 6, 113-141.

Bernsten, D. (2010). The unbidden past: Involuntary autobiographical memories as a basic mode of remembering. Current Directions in Psychological Science, 19, 138-142.

Brown, S. (2006). The perceptual music track: The phenomenon of constant musical imagery. Journal of Consciousness Studies, 13(6), 25-44.

Chin, T., \& Rickard, N. S. (2010). Non-performance, as well as performance, based music engagement predicts verbal recall. Music Perception, 27, 197-208.

Christoff, K., Ream, J. M., \& Gabrieli, J. D. E. (2004). Neural basis of spontaneous thought processes. Cortex, 40, 623-630.

Clark, L. A., \& Watson, D. B. (1995). Constructing validity: Basic issues in objective scale development. Psychological Assessment, 7, 309-319.

Collins, A. M., \& Loftus, E. E. (1975). A spreading-activation theory of semantic processing. Psychological Review, 82, 407-428.

Collins, A. M., \& Quillian, M. R. (1969). Retrieval time from semantic memory. Journal of Verbal Learning and Verbal Behavior, 8, 240-248.

Comrey, A. L. (1988). Factor-analytic methods of scale development in personality and clinical psychology. Journal of Consulting and Clinical Psychology, 56, 754-761.

Cottraux, J., Gérard, D., Cinotti, L., Froment, J. C., Deiber, M. P., Le Bars, D. et Al.(1996). A controlled positron emission tomography study of obsessive and neutral auditory stimulation in obsessive-compulsive disorder with checking rituals. Psychiatry Research, 60, 101-112.

Cuddy, L. L., Balkwill, L. L., Peretz. I., \& Holden, R. R. (2005). Musical difficulties are rare: A study of "tone deafness" among university students. Annals of the New York Academy of Science, 1060, 311-324.

DiStefano, C., Zhu, M., \& Mîndrilă, D. (2009). Understanding and using factor scores: Considerations for the applied researcher. Practical Assessment, Research and Evaluation, 14, 1-11.

Everitt, B., \& Hothorn, T. (2011). An introduction to applied multivariate analysis in $R$. New York: Springer.
Floridou, G., Williamson, V., \& Müllensiefen, D. (2012). Contracting earworms: The roles of personality and musicality. In E. Cambouropoulos, C. Tsougras, P. Mavromatis, \& K. Pastiadis (Eds.), Proceedings of the 12th International Conference on Music Perception and Cognition (ICMPC) (pp. 302-309). Athens, Greece: ICMPC / ESCOM. Retrieved from http://icmpc-escom2012.web.auth.gr/sites/default/files/papers/ 302_Proc.pdf

Foa, E. B., Huppert, J. D., Leiberg, S., Langner, R., Kichic, R., Наjсaк, G., \& Salkovskis, P. M. (2002). The obsessivecompulsive inventory: Development and validation of a short version. Psychological Assessment, 14, 485-496.

Fox, J. (2006). Structural-equation modeling with the sem package in R. Structural Equation Modeling, 13, 465-486.

Freeston, M. H., \& Ladouceur, R. (1997). What do patients do with their obsessive thoughts? Behaviour Research and Therapy, 35, 335-348.

Frost, R. O., Steketee, G., Cohn, L., \& Griess, K. (1994). Personality traits in subclinical and non-obsessive-compulsive volunteers and their parents. Behaviour Research and Therapy, $32,47-56$.

García-Soriano, G., Belloch, A., Morillo, C., \& Clark, D. A. (2011). Symptom dimensions in obsessive-compulsive disorder: From normal cognitive intrusions to clinical obsessions. Journal of Anxiety Disorders, 25, 474-482.

Gershunny, B. S., \& Sher, K. J. (1995). Compulsive checking and anxiety in a nonclinical sample: Differences in cognition, behavior, personality, and affect. Journal of Psychopathology and Behavioural Assessment, 17, 19-38.

GibBs, N. A. (1996). Nonclinical populations in research on obsessive-compulsive disorder: A critical review. Clinical Psychology Review, 16, 729-773.

Gönner, S., Hahn, S., Leonhart, R., Ecker, W., \& Limbacher, K. (2009). Identifikation der Hauptsymptome von Zwangspatienten anhand von Symptomskalen.

Kriteriumsvalidität und diagnostische Genauigkeit des OCI-R. [Identification of main symptoms in OCD patients by use of symptom scales. Criterion validity and diagnostic accuracy of the OCI-R]. Verhaltenstherapie, 19, 251-258.

Halpern, A. R., \& Bartlett, J. C. (2011). The persistence of musical memories: A descriptive study of earworms. Music Perception, 28, 425-432.

Halpern, A. R., \& Zatorre, R. (1999). When that tune runs through your head: A PET investigation of auditory imagery for familiar melodies. Cerebral Cortex, 9, 697-704.

Нickoк, G., Buchsbaum, B., Humphries, C., \& Muftuler, T. (2003). Auditory-motor interaction revealed by fMRI: Speech, music, and working memory in area Spt. Journal of Cognitive Neuroscience, 15, 673-682. 
Holm, S. (1979). A simple sequentially rejective multiple test procedure. Scandinavian Journal of Statistics, 6, 65-70.

Horn, J. L. (1965). A rationale and test for the number of factors in factor analysis. Psychometrika, 30, 179-185.

Hurlburt, R. T. (1979). Random sampling of cognitions and behavior. Journal of Research in Personality, 13, 103-111.

Kane, M. J., Brown, L. E., McVay, J. C., Silvia, P. J., Myin-Germeys, I., \& Kwapil, T. R. (2007). For whom the mind wanders, and when: An experience-sampling study of working memory and executive control in daily life. Psychological Science, 18, 614-621.

Kleber, B., Birbaumer, N., Veit, R., Trevorrow, T., \& Lotze, M. (2007). Overt and imagined singing of an Italian aria. Neuroimage, 36, 889-900.

Klinger, E. C., \& Cox, W. M. (1987). Dimensions of thought flow in everyday life. Imagination, Cognition and Personality, 7, 105-128.

Kraemer, D. J., Macrae, C. N., Green, A. E., \& Kelley, W. M. (2005). Musical imagery: Sound of silence activates auditory cortex. Nature, 434, 158.

Kvavilashvili, L., \& Mandler, G. (2004). Out of one's mind: A study of involuntary semantic memories. Cognitive Psychology, 48, 47-94.

Levitin, D. J. (2006). This is your brain on music. New York: Dutton.

Litkkanen, L. A. (2008). Music in everymind: Commonality of Involuntary Musical Imagery. In K. Miyazaki, Y. Hiragi, M. Adachi, Y. Nakajima, \& M. Tsuzaki (Eds.), Proceedings of the $10^{\text {th }}$ International Conference on Music Perception and Cognition (ICMPC 10) (pp. 408-412). Sapporo, Japan: ICMPC. Retrieved from http://i.org.helsinki.fi/lassial/files/publications/ 080904-Music_in_everymind_pdf.pdf

Liıkkanen, L. A. (2012). Musical activities predispose to involuntary musical imagery. Psychology of Music, 40, 236-256.

Loenlin, J. C. (2004). Latent variable models: An introduction to factor, path, and structural equation analysis (4th ed.) Mahwah, NJ: Lawrence Erlbaum.

MacDonald, A. M., \& De Silva, P. (1999). The assessment of obsessionality using the Padua inventory: Its validity in a British non-clinical sample. Personality and Individual Differences, 27, 1027-1046.

Mahendran, R. (2007). The psychopathology of musical hallucinations. Singapore Medical Journal, 48, 69-70.

Mataix-Cols, D., Cullen, S., Lange, K., Zelaya, F., Andrew, C., Amaro, E. Et Al. (2003). Neural correlates of anxiety associated with obsessive-compulsive symptom dimensions in normal volunteers. Biological Psychiatry, 53, 482-493.

Mataix-Cols, D., Junque, C., Sanchez-Turet, M., Vallejo, J., Verger, K., \& Barrios, M. (1999).

Neuropsychological functioning in a sub-clinical obsessivecompulsive sample. Biogical Psychiatry, 45, 898-904.
McVay, J. C., Kane, M. J., \& Kwapil, T. R. (2009). Tracking the train of thought from the laboratory into everyday life: An experience-sampling study of mind wandering across controlled and ecological contexts. Psychonomic Bulletin Review, 16, 857-863.

Müllensiefen, D., Gingras, B., Musil, J., Stewart, L. (2014). The musicality of non-musicians: An index for assessing musical sophistication in the general population. PLoS ONE, 9, e89642.

Rubenstein, C. S., Peynircioğlu, Z. F., Chambless, D. L., \& Piggot, T. A. (1993). Memory in sub-clinical obsessivecompulsive checkers. Behaviour Research and Therapy, 31, 759-765.

SACKs, O. (2007). Musicophilia: Tales of music and the brain. New York: Knopf.

SAlkovskis, P. M., \& Campbell, P. (1994). Thought suppression induces intrusion in naturally occurring negative intrusive thoughts. Behaviour Research and Therapy, 32, 1-8.

Sanchez, T., Rocha, S., Knobel, K., Kit, M., Santos, R., \& Pereira, C. (2011). Musical hallucination associated with hearing loss. Arquivos de neuro-psiquiatria, 69(2B), 395-400.

Saris, W. E., Satorra, A., \& Sorbom, D. (1987). The detection and correction of specification errors in structural equation models. In C. C. Clogg (Ed.), Sociological Methodology, (pp 105-129). San Francisco, CA: Jossey-Bass.

Sher, K. J., Frost, R. O., \& Oтto, R. (1983). Cognitive deficits in compulsive checkers: An exploratory study. Behaviour Research and Therapy, 21, 357-363.

Sher, K. J., Mann, B., \& Frost, R. O. (1984). Cognitive dysfunctions in compulsive checkers: Further explorations. Behaviour Research and Therapy, 22, 493-502.

Sorвom, D. (1989). Model modification. Psychometrika, 54, 371-384.

Sternberger, L. G., \& Burns, G. L. (1990). Maudsley obsessional compulsive inventory: Obsessions and compulsions in a non-clinical sample. Behaviour Research and Therapy, 28, 337-340.

Tolin, D., Abramowitz, J. S., Przeworski, A., \& FoA, E. B. (2002). Thought suppression in obsessive-compulsive disorder. Behaviour Research and Therapy, 40, 1255-1274.

Tulving, E., \& Schacter, D. L. (1990). Priming and human memory systems. Science, 247, 301-306.

Wammes, M., \& BARUšs, I. (2009). Characteristics of spontaneous musical imagery. Journal of Consciousness Studies, 16, 37-61.

Warner, N., \& Aziz, V. (2005). Hymns and arias: Musical hallucinations in older people in Wales. International Journal of Geriatric Psychiatry, 20, 658-660.

Wegner, D. M., Schneider, D. J., Carter, S. R., \& White, T. L. (1987). Paradoxical effects of thought suppression. Journal of Personality and Social Psychology, 53, 5-13. 
Wegner, D. M., \& Zanakos, S. (1994). Chronic thought suppression. Journal of Personality, 62, 615-640.

Werner, P., Swope, A., \& Heide, F. (2006). The Music Experience Questionnaire: Development and correlates. The Journal of Psychology, 140, 329-345.

Williamson, V. J., Jilka, S. R., Fry, J., Finkel, S., Müllensiefen, D., \& Stewart, L. (2012). How do earworms start? Classifying the everyday circumstances of Involuntary Musical Imagery. Psychology of Music, 40, 259-284.
Wu, K. D., \& Watson, D. (2002). Further investigation of the obsessive-compulsive inventory: Psychometric analysis in two non-clinical samples. Journal of Anxiety Disorders, 17, 305-319.

Zatorre, R. J., \& Halpern, A. R. (2005). Mental concerts: Musical imagery and auditory cortex. Neuron, 47, 9-12.

Zungu-Dirwayi, N., Hugo, F., van Heerden, B. B., \& Stein, D. J. (1999). Are musical obsessions a temporal lobe phenomenon? Journal of Neuropsychiatry and Clinical Neuroscience, 11, 398-400.

\section{Appendix A: Questionnaire items}

THE MUSICAL BEHAVIOR QUESTIONNAIRE

[Attend_Concert]

To the best of your memory, how many live concerts have you attended in the past 12 months?
1) None
2) $1-4$
3) $5-8$
4) $9-12$
5) 13 or more

[Attentive_Listening]

How often do you attentively listen to music (i.e. listening to the music is the main focus of your attention)?

1) Never 2) Once a week 3) A few times per week 4) Nearly every day

5) Once or twice a day 6) Several times per day

[Passive_Listening]

How often do you listen to music while you do other things (e.g. driving, working or exercising)?

1) Never 2) Once a week 3) A few times per week 4) Nearly every day

5) Once or twice a day 6) Several times per day

[Music_Importance_Life]

Thinking about all your hobbies and interests, how important is music to you?

$\begin{array}{lll}\text { 1) Not very important } & \text { 2) Somewhat important } & 3 \text { ) Very important 4) Extremely important }\end{array}$

[Musicianship]

How would you categorise yourself?

1) Non-musician 2) Music-loving non-musician 3) Amateur musician

4) Serious amateur musician 5) Semi-professional musician 6) Professional musician

[Singing_Ability]

Can you carry a tune (when singing)?

1) Not at all 2) Not very well 3) Fairly well 4) Very Well

[Singing_Along]

How often do you sing or hum along to music?

1) Never 2) Rarely 3) Some of the time 4) Often 5) Almost all the time

[Absolute_Pitch]

Do you have absolute pitch (e.g. can name a tone without a reference)?

1) Yes 2) No

[Play_Instrument]

Do you or have you ever played a musical instrument (includes voice)?

1) Yes 2) No

[Years_Musical_Activity]

How many years did you engage in sustained musical activity? years 
[Practice_Past]

Which category comes nearest to the amount of time you spent practicing an instrument (or voice) during that period of sustained musical activity?

1) About an hour per month 2) About an hour per week 3) About 15 minutes per day

4) About an hour per day 5) More than 2 hours per day

[Practice_Present]

Which category comes nearest to the amount of time you currently spend practicing an instrument (or voice)?

1) I rarely or never practise singing or playing an instrument anymore

2) About an hour per month 3) About an hour per week 4) About 15 minutes per day

5) About an hour per day 6) More than 2 hours per day

[Band_Play]

Have you ever participated in a musical group (e.g. band, choir, or orchestra)?

1) Yes 2) No

[Music_Exams]

Have you ever participated in musical competitions or examinations?

1) Yes 2) No

[Extra_Musical_Active1]

How many years have you engaged in musical training beyond that on instrument and voice

(e.g. Ear training, Harmony, Composition, Musical Analysis, Music history, Improvisation)? years

[Extra_Musical_Active2]

How many years training or practice have you engaged in for jobs that involve regular interaction with music (e.g., DJ, producer, sound engineer, tuner, etc.)? years

THE INVOLUNTARY MUSICAL IMAGERY QUESTIONNAIRE

* items are reverse scored

[InMI_Frequency]

On average I get earworms in my head*:

1) More than once a day 2) Once a day 3) At least once per week

4) At least once per month 5) Less than once per month

[InMI_Unpleasantness]

My earworms are:

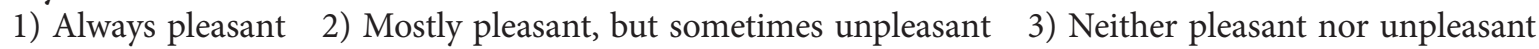

4) Mostly unpleasant, but sometimes pleasant

5) Always unpleasant

[InMI_Actively_Rid]

I actively try to get rid of my earworms*:

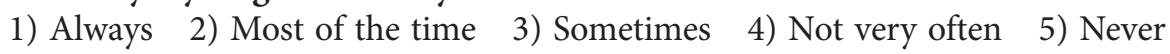

[InMI_Controlability]

To get rid of an earworm is:

$\begin{array}{lll}\text { 1) Easy and takes no effort } & \text { 2) Easy but takes some effort } & \text { 3) Sometimes hard }\end{array}$

4) Nearly always very hard 5) Impossible 6) N/A - I never try to get rid of earworm 
[InMI_Length]

On average, my earworms normally last:

1) Less than 10 minutes 2) Between 10 minutes and half an hour

3) Between half an hour and 1 hour 4) Between 1 and 3 hours 5) More than 3 hours

[InMI_Interference]

I find my earworms:

1) Never interfere with other things I am doing 2) Interfere a little with other things I am doing

3) Sometimes interfere with other things I am doing 4) Often interfere with other things I am doing

5) Always interfere with other things I am doing

[InMI_Feeling]

Does it worry you when an earworm is stuck in your head?

1) Not at all 2) A little 3) Sometimes 4) Often 5) Very frequently 6) Always

Appendix B: Additional tables and figures

APPENDIX B, TABLE 1. Summary statistics of items from Musical Behavior and INMI questionnaire as well as OCI-R.

\begin{tabular}{|c|c|c|c|c|}
\hline Items & $M$ & $S D$ & Skewness & Kurtosis \\
\hline \multicolumn{5}{|l|}{ Music Bevahior Questionnaire } \\
\hline Amount of Instrumental Practice (Past) & 2.58 & 1.52 & -0.46 & 2.17 \\
\hline Amount of Instrumental Practice (Present) & 3.47 & 2.34 & -0.21 & 1.51 \\
\hline Self-rated Musicianship & 2.74 & 1.22 & 0.78 & 3.19 \\
\hline Years of Sustained Musical Activity & 9.06 & 10.43 & 1.98 & 7.51 \\
\hline Musical Training Beyond Instrument & 1.75 & 4.93 & 4.70 & 31.62 \\
\hline Training Music-Related Jobs & 1.52 & 5.26 & 4.81 & 29.59 \\
\hline Amount of Attentive Listening & 3.82 & 1.58 & -0.04 & 1.86 \\
\hline Importance of Music in Life & 3.11 & 0.99 & -0.58 & 2.28 \\
\hline Amount of Passive Listening & 4.87 & 1.29 & -0.80 & 2.61 \\
\hline Concert Attendance & 2.49 & 1.22 & 0.79 & 2.71 \\
\hline Self-rated Singing Ability & 2.89 & 0.94 & -0.47 & 2.32 \\
\hline Extent of Sing-along Behavior & 3.80 & 2.79 & -0.55 & 2.79 \\
\hline \multicolumn{5}{|l|}{ INMI Questionnaire } \\
\hline Interference of INMI Experiences & 2.00 & 0.82 & 0.49 & 2.92 \\
\hline Feeling worried about INMI Experiences & 1.49 & 0.84 & 1.76 & 6.08 \\
\hline Active Attempts to Get Rid of INMI Experiences & 2.52 & 0.85 & 0.21 & 3.12 \\
\hline Effort to Control INMI Experiences & 3.00 & 1.00 & -0.89 & 2.63 \\
\hline Length of INMI episodes & 2.79 & 1.35 & 0.27 & 1.84 \\
\hline Frequency of INMI Episodes & 3.55 & 1.13 & -0.30 & 2.38 \\
\hline Unpleasantness of INMI Experiences & 2.07 & 0.77 & 0.71 & 3.88 \\
\hline \multicolumn{5}{|l|}{ OCI-R } \\
\hline Hoarding & 3.77 & 2.71 & 0.71 & 3.01 \\
\hline Checking & 2.33 & 2.40 & 1.46 & 5.16 \\
\hline Ordering & 3.13 & 2.73 & 1.01 & 3.64 \\
\hline Neutralising & 1.41 & 2.10 & 2.11 & 7.94 \\
\hline Washing & 1.07 & 1.93 & 2.44 & 9.75 \\
\hline Obsessing & 2.76 & 2.86 & 1.20 & 3.95 \\
\hline
\end{tabular}

Binary Variables

Proportion of Yes Responses

Ever Played Instrument

0.81

Ever Played in Band / Ensemble $\quad 0.68$

Ever Taken Music Exams $\quad 0.50$

$\begin{array}{ll}\text { Have Absolute Pitch } & 0.10\end{array}$

Note. Summary statistics are based on the full sample of $N=1536$ participants. 
338 Daniel Müllensiefen, Joshua Fry, Rhiannon Jones, Sagar Jilka, Lauren Stewart, \& Victoria J Williamson

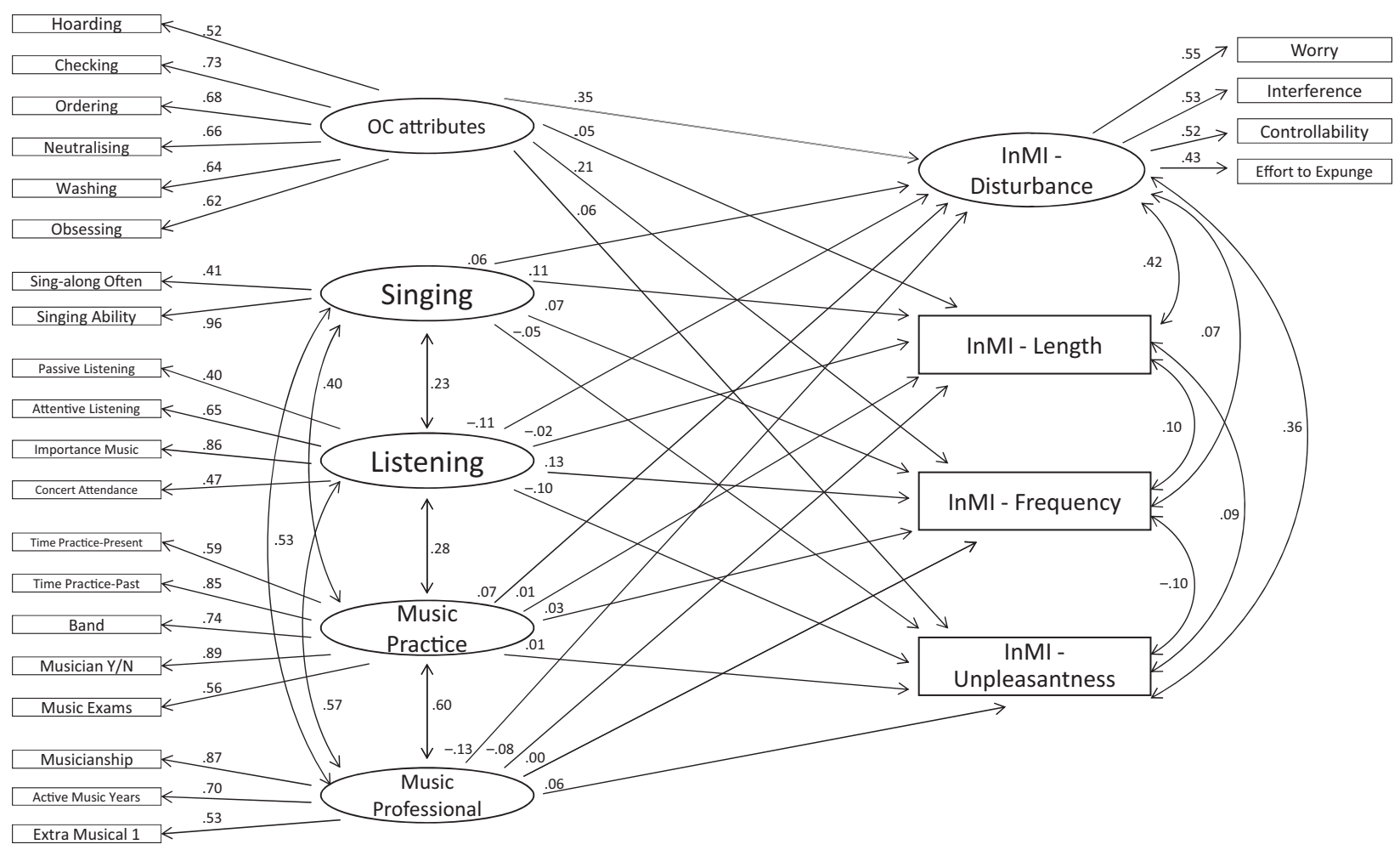

APPENDIX B, FIGURE 1. Graphical representation of full structural equation model. 